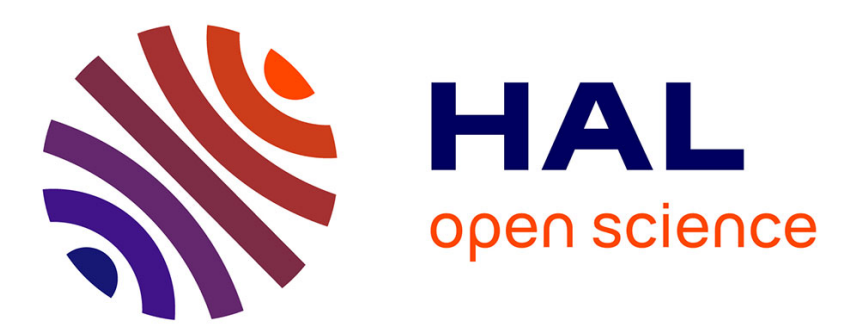

\title{
Le patient peut-il et doit-il participer à la gestion des risques en radiothérapie?
}

Vanina Mollo, Adeline Pernet Pernet, Grégoire Moutel, Nathalie Duchange, Philippe Giraud

\section{- To cite this version:}

Vanina Mollo, Adeline Pernet Pernet, Grégoire Moutel, Nathalie Duchange, Philippe Giraud. Le patient peut-il et doit-il participer à la gestion des risques en radiothérapie?: Le patient et le risque en radiothérapie. Cancer/Radiothérapie, 2011, 15 (3), pp.176-81. 10.1016/j.canrad.2010.09.003. inserm-00557208

\section{HAL Id: inserm-00557208 https://www.hal.inserm.fr/inserm-00557208}

Submitted on 18 Jan 2011

HAL is a multi-disciplinary open access archive for the deposit and dissemination of scientific research documents, whether they are published or not. The documents may come from teaching and research institutions in France or abroad, or from public or private research centers.
L'archive ouverte pluridisciplinaire HAL, est destinée au dépôt et à la diffusion de documents scientifiques de niveau recherche, publiés ou non, émanant des établissements d'enseignement et de recherche français ou étrangers, des laboratoires publics ou privés. 


\title{
Le patient peut-il et doit-il participer à la gestion des risques en
}

\section{radiothérapie ?}

\section{Can or must the patient participate to risk management in radiotherapy?}

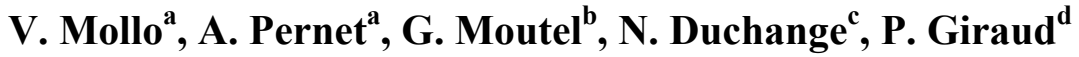 \\ ${ }^{\text {a }}$ Laboratoire d'ergonomie, Centre de recherche sur le travail et le développement (CRTD - \\ EA 4132), Conservatoire national des arts et métiers (Cnam), 41 rue gay Lussac, 75005 Paris. \\ ${ }^{\mathrm{b}}$ Université Paris Descartes, Unité de médecine sociale et d'éthique médicale, Hôpital \\ Corentin Celton, 4 parvis Corentin-Celton, 92133 Issy-les-Moulineaux cedex. \\ ${ }^{\mathrm{c}}$ Université Paris Descartes, Laboratoire d'éthique médicale et de médecine légale, 45 rue des \\ St Pères, 75006 Paris. \\ ${ }^{\mathrm{d}}$ Université Paris Descartes, service d'oncologie radiothérapie, Hôpital Européen Georges \\ Pompidou (HEGP), 20 rue Leblanc, 75015 Paris.
}

Titre court : Le patient et le risque en radiothérapie.

Correspondant :

Vanina Mollo

Centre de recherche sur le travail et le développement (CRTD - EA 4132)

Conservatoire national des arts et métiers (Cnam)

41, rue gay Lussac

75005 Paris

Téléphone: (33) 144107868

Fax: (33) 144107934

Courriel: vanina.mollo@cnam.fr 


\section{Résumé}

Objectif. Cette étude avait pour objectif d'analyser si la participation des patients à la gestion des risques est une voie d'amélioration possible de la sécurité des soins en radiothérapie, ou pas. Elle repose sur l'hypothèse qu'une telle participation permet de développer une culture de sécurité axée sur la coopération soignant/soigné, d'améliorer la satisfaction des patients, et de réduire les coûts associés aux événements indésirables.

Matériel et méthodes. Un atelier a été organisé dans le cadre d'une formation des professionnels de la radiothérapie à la gestion des risques. Les professionnels étaient d'abord répartis en trois sous-groupes selon la spécialité (médecins, physiciens médicaux et manipulateurs), et devaient travailler sur quatre grandes questions relatives à la participation, dont le recueil d'expériences vécues dans lesquelles les patients ont contribué (positivement ou négativement) à la sécurité des soins. Les résultats ont ensuite été restitués collectivement. Résultats. La participation des patients permet non seulement de détecter et de récupérer certains oublis ou erreurs de la part des professionnels (oubli du cache, erreur d'identité), mais aussi de diminuer les comportements à risque des patients (prendre volontairement la place d'un autre patient pour passer plus vite). Cependant, cela doit être une possibilité offerte au patient, et non une obligation.

Conclusion. La participation des patients à la sécurité des soins est un champ d'étude qui nécessite d'être développé afin de définir les conditions favorables à une telle participation, et d'implémenter une série d'actions visant à améliorer la sécurité par une gestion coopérative de celle-ci.

Mots clés. Participation des patients, sécurité des soins, radiothérapie. 


\begin{abstract}
Purpose. The present study aimed at analyzing if patient participation constitutes a promising way of improvement of patient safety, or not. The hypothesis is that patient participation is a means to develop a safety culture based on the cooperation between patients and healthcare providers, to improve patients' satisfaction and to reduce the costs associated to adverse events.
\end{abstract}

Materials and methods. A half-day session was organized on this theme during a training of radiotherapy professionals on risk management. Professionals were first distributed in three subgroups according to their speciality (radiation oncologists, radiation physicists and medical technicians), and had to work on four main questions relating to participation, among which the collection of real situations in which patients effectively contributed (positively or negatively) to patient safety. Results were then collectively discussed.

Results. Patient participation allows not only to detect and recover some mistakes or errors made by the professionals (error of identity), but also to decrease patients' risk behaviours (take voluntarily the place of another patient in order to be treated faster). However, it must be seen as a possibility offered to patients, and not as an obligation.

Conclusion. Patient participation to patient safety is a field of study which requires to be developed in order to define the conditions enhancing such participation and to implement a set of actions to improve healthcare safety by a cooperative management of this one.

Keywords. Patient participation, healthcare safety, radiotherapy. 


\section{Introduction}

La sécurité des soins est devenue une priorité centrale pour les politiques publiques, tant au niveau national qu'européen. En raison des accidents d'irradiation récents d'Epinal et de Toulouse, la radiothérapie est une des premières spécialités médicales qui a du intégrer très rapidement dans son fonctionnement une culture du risque. Les patients soumis à un traitement par radiothérapie s'engagent dans un processus long et complexe où interviennent plusieurs professionnels. Du fait de la répétition des séances de traitement, ils acquièrent une certaine connaissance du déroulement de chaque séance qui leur permet potentiellement de jouer un rôle actif dans la gestion des risques. Cette position d'acteur demeure cependant peu explorée en France. Cet article analyse si et sous quelles formes les patients peuvent contribuer à améliorer la sécurité des soins en radiothérapie.

Après avoir présenté le contexte législatif en faveur d'une participation active des patients, et défini ce qu'on entend par participation des patients à la sécurité des soins, nous présenterons les résultats d'un atelier mené lors d'une formation des différents personnels de radiothérapie à la gestion des risques. Cet atelier avait pour objectif de réfléchir sur la question de la participation des patients à la sécurité des soins, et était fondé sur des échanges d'expériences entre les différents professionnels.

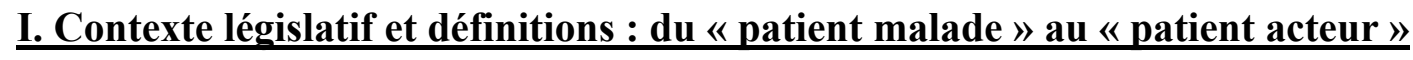

\section{I.1 Une évolution légiférée de la place des patients dans le système de santé}

Favoriser l'implication des patients correspond à la volonté de l'Union Européenne de développer des structures permettant aux patients de participer aux processus de décision concernant leurs soins de santé. Parmi ses recommandations, l'Union Européenne met l'accent sur la nécessité, pour les états membres, d'autonomiser et d'informer les patients. Ceci passe par : 
- l'intégration de représentants de patients à l'élaboration des politiques et des programmes de sécurité des patients à tous les échelons ;

- l'information des patients quant aux niveaux de sécurité et aux mesures en vigueur pour réduire ou éviter les erreurs et pour garantir un consentement éclairé au traitement, faciliter les choix et les décisions du patient;

- l'instauration ou le renforcement des systèmes de signalement des événements indésirables capables de tirer des enseignements des défaillances, qui offrent la possibilité aux patients, à leur famille et à d'autres proches soignants de faire part de leur expérience.

En France, c'est en 2002 qu'une avancée majeure est intervenue avec la loi n ${ }^{\circ} 2002-303$ du 4 mars « relative aux droits des malades et à la qualité du système de santé », publiée au Journal Officiel du 5 mars 2002. La participation est alors vue comme un moyen d'améliorer le fonctionnement des systèmes de santé. Au plan institutionnel, il y a désormais des structures qui permettent cette participation comme les conseils régionaux de santé, le collectif inter-associatif sur la santé, le Haut Conseil de la Santé Publique, les Commissions des Relations avec les Usagers et de la Qualité de la prise en charge (CRUQ), etc. Au niveau de la relation médecin-patient, la loi rappelle l'obligation déontologique de faire participer le patient à la décision et de l'impliquer dans la démarche de soins. Ainsi comme le souligne 1'article L.1111-2 du Code de la Santé Publique, reprenant les termes de la loi du 4 mars 2002 relative aux droits des malades et à la qualité du système de santé, « Toute personne a le droit d'être informée sur son état de santé. Cette information porte sur les différentes investigations, traitements ou actions de prévention qui sont proposés, leur utilité, leur urgence éventuelle, leurs conséquences, les risques fréquents ou graves normalement prévisibles qu'ils comportent ». Toutefois, l'article précise que «la volonté d'une personne d'être tenue dans l'ignorance d'un diagnostic ou d'un pronostic doit être respectée ». Dans le même esprit, 
l'article 35 du Code de Déontologie Médical (version du 8 août 2004) souligne que «Le médecin doit à la personne qu'il examine, qu'il soigne ou qu'il conseille une information loyale, claire et appropriée sur sn état, les investigations et les soins qu'il lui propose. Tout au long de la maladie, il tient compte de la personnalité du patient dans ses explications et veille à leur compréhension. Toutefois, dans l'intérêt du malade et pour des raisons légitimes que le praticien apprécie en conscience, un malade peut être tenu dans l'ignorance d'un diagnostic ou d'un pronostic graves ». Ainsi la participation du patient à ses soins s'impose de plus en plus, mais il faut avoir conscience que ce principe mérite modulation et prudence en fonction du contexte clinique, ce qui inclue la gravité de la maladie, l'âge et le niveau de conscience. Comme le souligne Thouvenin, le modèle français repose sur la concertation et le fait que «les propositions faites par le professionnel de santé soient l'objet d'une discussion au terme de laquelle une décision conjointe sera prise » [1].

Ces éléments soulignent les évolutions majeures de notre pays en matière de participation des patients. Cependant, les liens entre participation des patients et sécurité des soins sont aujourd'hui peu établis en France [2]. Les approches poursuivies en matière de gestion des risques sont essentiellement prescriptives (accroissement des procédures et des réglementations) et prennent insuffisamment en compte les savoirs de fiabilité acquis dans d'autres secteurs (aéronautique, nucléaire et industries à risque), ainsi que les modèles développés par l'économie des services, qui insistent sur la coopération avec les usagers, c'est-à-dire sur la participation de ces derniers à la réalisation du service. En médecine, faire participer le patient à sa propre sécurité mérite d'être étudiée en terme d'efficacité, mais aussi en terme éthique en fonction des profils de patients.

\section{I.2 Définitions : la participation des patients à la sécurité des soins}

La participation des patients à la sécurité des soins peut être définie comme les actions qu'ils peuvent mettre en œuvre pour réduire la probabilité d'erreurs médicales et/ou pour atténuer 
les effets des erreurs lorsqu'elles surviennent effectivement [3]. Les raisons qui justifient une plus grande participation des patients à la sécurité des soins sont nombreuses. Premièrement, ils sont au cœur du processus de soin, non seulement parce qu'ils sont les uniques bénéficiaires du soin, mais aussi parce qu'ils peuvent observer quasiment l'ensemble du processus de soin $[3,4]$, qui s'étend de la consultation initiale aux soins à domicile. Cela leur permet de détecter et de signaler des erreurs éventuelles, et donc de contribuer à l'amélioration de la sécurité. Deuxièmement, tout comme les professionnels de santé, les patients eux aussi peuvent commettre ou être à la source des erreurs [5]. Cela constitue une raison supplémentaire de leur permettre de jouer un rôle actif dans la gestion de la sécurité. C'est un moyen d'améliorer la prise de conscience des patients et des professionnels de santé à propos de la sécurité des soins, et de réduire la probabilité d'erreurs par une gestion coopérative de celles-ci. Troisièmement, l'accès à l'information médicale est facilité par les médias et Internet, et cela impacte la relation entre les professionnels de santé et les patients; ces derniers étant plus exigeants sur certains aspects de leur soin. En dépit de la nécessité de contrôler la qualité des connaissances accessibles sur le Net, le niveau grandissant de connaissance des patients amène à penser que ces derniers sont de plus en plus capables de jouer un rôle actif dans la gestion de la sécurité des soins : en étant mieux informés sur le processus de soin, ils sont plus à même de détecter des événements indésirables ou inhabituels.

L'étude exploratoire décrite ci-après présente une série de situations dans lesquelles les patients peuvent contribuer effectivement à la gestion de la sécurité des soins en radiothérapie. Elle discute également des conditions à développer pour une plus grande participation des patients, mais aussi des limites et obstacles que cela soulève, notamment en 
termes de responsabilité et de vécu psychologique de leur part et de la part des équipes soignantes.

\section{Atelier}

\section{II.1 Participants et Méthode}

La présente étude s'inscrit dans le cadre d'une collaboration initiée depuis plus d'un an entre les Sociétés Savantes des oncologues radiothérapeutes (SFRO) et des physiciens médicaux (SFPM) ainsi qu'avec l'Institut de Radioprotection et de sûreté nucléaire (IRSN), l'Autorité de Sûreté Nucléaire (ASN) et le Conservatoire national des arts et métiers (Cnam), et dédiée à la formation des différents personnels de radiothérapie à la gestion des risques. Lors de la préparation de cette formation, il est apparu qu'un acteur essentiel manquait dans la présentation habituelle de la gestion des risques : le patient lui-même. Le patient peut-il participer activement à sa propre sécurité ? Le faut-il ? Comment? Ces questions ont fait l'objet d'une réflexion sous forme d'atelier dans cette formation.

L'atelier s'est tenu lors de la première session de formation à la gestion des risques organisée par l'Ecole de Formation Européenne en Cancérologie (EFEC) sous l'égide de la SFRO, la SFPM, l'IRSN et l'ASN à l'hôpital européen Georges Pompidou du 9 au 11/09/2009. Elle s'est déroulée sur deux heures, réparties comme suit: 20 minutes d'introduction et de présentation du contexte législatif en matière de participation des patients (phase 1); 45 minutes de travaux dirigés par groupe de professionnels (phase 2); 30 minutes de restitution des résultats et discussion (phase 3); 25 minutes de conclusion et de mise en perspective des résultats par rapport aux travaux actuels (revue de littérature internationale) (phase 4). Seules les phases 2 et 3 sont détaillées ici.

Les professionnels devaient remplir un formulaire comportant les quatre questions suivantes : 
- Décrivez des exemples que vous avez vécus (vous personnellement, de préférence) ou qui vous ont été rapportés par vos collègues, dans lesquels le patient est intervenu de façon positive / négative dans la sécurité des soins ;

- Citez les initiatives mises en place dans vos établissements pour encourager l'implication des patients à la gestion de leurs propres soins, et/ou la participation des patients à la sécurité des soins ;

- Citez les besoins et/ou outils à mettre en place selon vous pour favoriser la participation des patients à la sécurité des soins ;

- Citez les limites ou obstacles à la participation des patients à la sécurité des soins.

Les professionnels étaient répartis en trois sous-groupes, en fonction de leurs spécialités : un groupe d'oncologues radiothérapeutes, un groupe de physiciens médicaux, et un groupe de manipulateurs. Cette répartition avait pour but d'analyser si les résultats différaient ou non en fonction de la spécialité, et de faciliter les échanges d'expériences au cours de la restitution. Les résultats se limiteront à une description qualitative.

\section{II.2 Résultats}

Après avoir décrit les exemples relatés par les professionnels, les résultats présenteront les initiatives qui selon les professionnels permettent une implication active des patients dans le processus de soin, ainsi que les besoins qui restent à satisfaire. Les obstacles et limites à une participation active des patients seront ensuite discutés.

\section{$\underline{\text { II.2.1 Exemples }}$}

Au total, 17 exemples ont été rapportés.

Quatorze exemples relatent des situations dans lesquelles les patients participent activement et efficacement à la sécurité des soins. Ils sont détaillés dans le tableau I. La plupart du temps 
(10/14), il s'agit de situations dans lesquelles les patients signalent des oublis ou erreurs éventuelles de la part des professionnels de santé. Par exemple, le patient signale l'oubli d'un cache, une erreur d'identité, rectifie la partie ou le côté du corps à irradier, signale qu'un faisceau n'a pas été traité. Les manipulateurs déclarent à ce propos « qu'il y a au moins 10\% d'erreurs qui sont évitées simplement parce que le patient nous dit Est ce que vous n'avez pas oublier de mettre un filtre? Est ce que ceci? Est ce que cela? Ils sont attentifs du moment où ils arrivent jusqu'à ce qu'ils partent, chacun à son niveau, chacun sur un point particulier. Ils savent ce qu'on fait, ce qu'on devrait leur faire, et ce qu'on oublie de leur faire ». Parmi les 4 autres exemples, 3 concernent des patients qui signalent des événements inhabituels, c'est-àdire des écarts et/ou changements par rapport aux procédures de traitement habituelles. Ces déclarations peuvent révéler des oublis ou erreurs effectifs, mais pas toujours. Par exemple, le patient peut signaler une différence dans la durée de traitement, mais qui s'explique par la réalisation d'un complément de radiation ou d'un contrôle de positionnement. La dernière situation relate un patient qui écoute de la musique pour se relaxer et ainsi éviter de bouger lors de l'irradiation.

Les trois exemples suivants relatent des situations où l'intervention du patient engendre un risque pour la sécurité des soins, qu'il s'agisse de sa propre santé ou de celle d'autres patients (cf. tableau II). Un exemple cité à deux reprises est celui du patient qui prend volontairement la place d'un autre patient, en déclinant une fausse identité, afin d'être traité plus rapidement. La conséquence est la réalisation du mauvais traitement si l'équipe soignante n'est pas vigilante.

\section{$\underline{\text { II.2.2 Initiatives }}$}


$\mathrm{Au}$ total, les professionnels ont rapporté 10 initiatives mises en place dans leurs établissements pour encourager l'implication des patients à la gestion de leurs propres soins, et/ou la participation des patients à la sécurité des soins. Il ne s'agit pas d'une liste exhaustive, mais des actions jugées les plus représentatives par les professionnels. Elles peuvent être divisées en 3 grandes catégories.

La première catégorie concerne les actions d'information à destination des patients, et regroupe 6 initiatives. Elle comprend les sites Internet mis en place par les différents services, le programme personnalisé de soins détaillant le circuit patient, la maison des usagers, la présence de représentants d'associations de patients dans les salles d'attente de traitement, les notes d'information relatives à la sécurité (maintenance des machines, contrôles qualité...). Cette catégorie comprend également le contrôle de la qualité des informations par les patients eux-mêmes.

La seconde catégorie concerne la gestion de la sécurité des soins, et comprend deux types d'initiatives différentes. La première renvoie directement à la participation des patients à la sécurité des soins, et consiste en l'autocontrôle par le patient de sa propre identité. Elle se divise en deux actions rapportées par les établissements :

- l'affichage de la photo du patient en salle de traitement. Le patient doit alors vérifier que la photo lui correspond bien ;

- le passeport d'identité qui contient les nom, prénom, photo et date de naissance du patient. Ce passeport est conservé par le patient, et vérifié par les manipulateurs à chaque séance.

La seconde initiative concerne les professionnels de santé, et plus précisément la gestion du Workflow dans le processus de traitement du patient. Elle comprend les cahiers de liaison avec les chimiothérapeutes pour prévenir les éventuels soucis d'hospitalisation, ou pour informer les oncologues qui ne pratiquent pas dans l'établissement en question. 
La troisième catégorie, quand à elle, concerne l'implication des patients dans la gestion des soins, et regroupe 2 initiatives. Elle comprend l'intégration des patients dans les différentes commissions des établissements (CRUQ, commission de surveillance, commission des relations avec les usagers et de la qualité de la prise en charge...), et dans l'évaluation de la qualité des soins prodigués (questionnaires de satisfaction, espaces de discussion sur les sites des services...).

\section{$\underline{\text { II.2.3 Besoins et/ou outils }}$}

En dépit des initiatives mises en place dans les établissements, les professionnels ont exprimés trois grands types de besoins et/ou outils qui demeurent non satisfaits.

Les premiers concernent l'information des patients. Ils comprennent à la fois les supports d'information existants (livrets d'accueil, programme personnalisé de soins, fiches d'informations...) mais aussi les points à améliorer et/ou à développer pour encourager la participation au travers de l'information. Par exemple : accorder plus de temps au patient pour qu'il se rende compte de ce qu'on va lui faire et pour qu'il parle ; favoriser et généraliser les consultations d'annonce en radiothérapie pour expliquer le traitement et l'encourager à participer.

Quelques fois les professionnels n'avaient pas la même perception des informations utiles à communiquer au patient. L'extrait suivant reprend un échange entre un manipulateur (M), qui souligne la nécessité d'informer les patients sur le fait qu'ils peuvent participer, et un radiothérapeute $(\mathrm{R})$ qui associe information et responsabilisation du patient :

« M : Le patient a déjà un degré de connaissances quant même... il suffit de lui dire signalez-nous si vous voyez quelque chose d'anormal...

$\mathrm{R}$ : Ca peut être anxieux pour pas mal de patients. Où est ce qu'on met les limites entre l'information et la responsabilisation? 
M: Ce n'est pas le responsabiliser que de lui dire... c'est simplement lui dire très gentiment: nous des patients on en traite 50,60 par jour et donc on peut un jour oublier quelque chose donc s'il vous plait si vous vous rendez compte que quelque chose est différent, dîtes le nous.

$\mathrm{R}$ : Oh, non.

M : Eh ben si au niveau de la confiance c'est quelque chose d'essentiel quand un patient sait qu'il a le droit de faire remarquer. Parce que des patients [non informés] ne vont pas oser, ils vont le dire un, deux ou trois jours après. Vous pouvez simplement leur demander ça, pas à tous les patients, mais pour beaucoup il n'y a aucun souci ».

Cet extrait illustre comment, dans l'interaction, l'information donnée au patient pourrait avoir des conséquences favorables pour améliorer la sécurité des soins.

Les seconds renvoient à la formation des professionnels. Ceux-ci ont en effet soulignés qu'ils n'étaient pas formés à la gestion de la relation avec les patients. Ils ont notamment exprimés un besoin de formation à l'écoute active afin d'être plus attentifs et réactifs aux interventions des patients, de mieux les engager dans les décisions et de distinguer parmi différentes situations celles qui requièrent leur participation. Ils ont également exprimé un besoin de formation à l'information : comment informer à l'aide de concepts simples ? Quelle information donner? Sous quelle forme ? etc.

Les derniers englobent des outils de gestion des risques, comme la mise en place de systèmes d'identification du patient (carton de rendez-vous avec photo, systèmes électroniques), et des moyens permettant d'impliquer plus directement le patient dans la gestion de son propre traitement, comme par exemple : le positionnement aidé par le patient ; la réalisation d'une séance «à blanc» pour habituer le patient au matériel, pour qu'il soit moins angoissé et plus actif dans le traitement, etc. 


\section{$\underline{\text { II.2.4 Obstacles et limites }}$}

Quatre obstacles et limites à la participation des patients à la sécurité des soins ont été relevés par les professionnels de santé.

Les premiers concernent le stress et l'angoisse des patients qui peuvent compromettre leurs capacités d'écoute et de compréhension, mais aussi des professionnels eux-mêmes qui associent participation et responsabilisation des patients.

Les seconds concernent l'information des patients. Elle comprend d'une part, la fiabilité des informations dont disposent les patients (en particulier lorsqu'ils utilisent Internet) qui peut les conduire à des jugements erronés ou inadaptés, d'autre part le discours du patient sur son histoire clinique, qui peut ne pas être fiable, et enfin la nature des informations à donner aux patients et au langage utilisé pour les transmettre (un langage trop technique n'étant pas accessible pour les patients).

Les troisièmes renvoient à la diversité des patients, (en termes d'âge, de culture, de niveau d'éducation, de personnalité) qui influe sur leur degré de participation. Les professionnels ont notamment soulignés les problèmes de langues qui peuvent limiter les échanges, la personnalité des patients qui impacte la volonté de participation des patients mais aussi la capacité des professionnels à impliquer les patients, ainsi que la diversité interculturelle et intergénérationnelle qui ne permettent pas une standardisation de la relation soignant-soigné.

Les quatrièmes, quant à eux, concernent l'organisation du travail, et en particulier la disponibilité temporelle des professionnels. En effet, favoriser la participation implique d'accorder un temps plus important à l'échange professionnel - patient. Cette condition est en contradiction avec les conditions de travail actuelles des professionnels de santé, qui réduisent le temps que ces derniers peuvent consacrer au dialogue avec le patient.

\section{Discussion}


Il existe dans les établissements de soins une importante variété d'actions à destination des patients. La plupart sont centrées sur l'information. Elles permettent de s'assurer que les patients comprennent leur maladie et les traitements associés, et de les accompagner tout au long de leur prise en charge pour répondre à leurs divers besoins. Elles dotent également les patients de compétences relatives à leur santé, et ainsi leur offrent la possibilité de participer plus activement aux décisions et actions les concernant, en partenariat avec les professionnels. En revanche, peu d'initiatives concernent directement la participation des patients à la sécurité des soins. Pourtant, les expériences relatées par les professionnels lors de cette formation montrent que la participation des patients est une voie possible d'amélioration de la sécurité des soins en radiothérapie. En effet, à travers leurs interventions, les patients peuvent participer à la détection et à la récupération d'oublis ou erreurs. De même, en étant mieux impliqués, ils sont plus à même de comprendre les risques associés aux soins, ce qui permet d'éviter certains comportements à risque. Par exemple, un patient peut prendre la place d'un autre en pensant que tous les patients reçoivent le même traitement de radiothérapie, ou que les traitements diffèrent mais que les écarts de dose sont sans conséquence.

Ces éléments amènent à réfléchir sur l'intérêt de compléter les méthodologies existantes de gestion des risques par la prise en compte des initiatives patients [2], par exemple en offrant la possibilité aux patients ainsi qu’à leur famille de déclarer des événements indésirables [6].

Développer la participation implique un renforcement des capacités des patients de façon à leur permettre de participer activement à la gestion de leurs soins [7]. Ceci repose sur au moins trois conditions. D'une part, l'information et la formation des patients. Comme le souligne Amalberti, l'éducation thérapeutique est un outil essentiel pour renforcer les compétences de compréhension des patients et leur rôle actif dans l'auto-surveillance des risques associés aux maladies graves et chroniques [8]. D'autre part, la formation des professionnels de santé à 
cette thématique afin de renforcer leurs capacités d'écoute et d'information des patients, et de développer une relation de partenariat. Ce partenariat n'a d'ailleurs rien d'évident dans un contexte professionnel marqué par une culture d'expertise médicale [9,10], mais il s'agit d'une condition nécessaire pour promouvoir une gestion participative des soins en santé. Enfin, les conditions précitées supposent un effort délibéré dans les politiques de santé afin qu'une telle participation informée soit possible.

La notion même de participation est difficilement dissociable de celle de responsabilité. En effet, faire participer plus activement les patients à la gestion des risques signifie les responsabiliser afin de devenir acteurs de leurs propres soins. Cependant, la notion de responsabilité telle qu'entendue ici ne doit pas réduire le niveau et l'exigence de responsabilité des professionnels : en effet au plan médico-légal, la sécurité des soins incombe encore à ce jour indiscutablement aux professionnels de santé et aux institutions où ils travaillent. On ne peut attribuer au patient qu'un niveau d'expertise complémentaire qui doit être doublée par celle d'un professionnel : ainsi les professionnels disposent de l'expertise clinique et technique pour définir et réaliser les traitements. Les patients quant à eux peuvent être en mesure d'évaluer l'application et les effets secondaires et de signaler tout changement ou incompréhension dans le cours de réalisation de leur traitement.

Ces éléments mettent l'accent sur la nécessité de développer l'éducation thérapeutique des patients, définie comme « un ensemble de pratiques visant à permettre au patient l'acquisition de compétences, afin de pouvoir prendre en charge de manière active sa maladie, ses soins et sa surveillance, en partenariat avec ses soignants ». ${ }^{1}$ Mais pour cela il est nécessaire de former les professionnels de santé eux-mêmes à l'éducation des patients, afin de mettre ces derniers en capacité de participer plus activement à la gestion des risques. Au plan déontologique, il

\footnotetext{
${ }^{1}$ http://www.sante.gouv.fr/htm/pointsur/eduthera/notinte.htm\#1a
} 
faut cependant veiller à ne pas impliquer systématiquement et sans discernement les patients dans le suivi de leurs soins. Si pour certains cela peut apparaître positif, le code de déontologie rappelle que la démarche de soins et l'implication du patient, incluant son information, doivent être modulées en fonction du contexte clinique. Ainsi l'état psychique, 1'âge, les différences culturelles, et la gravité de la maladie sont à prendre en compte.

Ainsi, la participation des patients à la sécurité des soins ne peut et ne doit pas être une injonction systématique [11]. Il est donc difficile de définir des procédures standardisées concernant le rôle que les patients doivent jouer dans la gestion de leur propre sécurité [12]. Trois idées sous-tendent cette affirmation.

Premièrement, la volonté de participation varie selon les patients, essentiellement en raison de ce que ce concept signifie pour eux, et du vécu psychologique de la maladie. En effet, certains patients désirent être acteurs de leur soin, alors que d'autres préfèrent un rôle plus passif. Ces derniers risquent d'être déstabilisés si cette participation est vécue comme une charge supplémentaire à leur maladie.

Deuxièmement, prendre en compte que la participation du patient peut être pour lui anxiogène. C'est la raison pour laquelle il peut sembler inenvisageable pour certaines équipes de demander au patient de "surveiller" lui-même le travail qu'ils effectuent sous peine de déclencher une grave crise de confiance. Ceci nécessite donc un apprentissage d'un discours professionnel permettant de ne pas générer d'anxiété et de bien accompagner un patient dans cette démarche.

Troisièmement, le niveau de participation des patients peut varier en fonction de la gravité des tableaux cliniques (certaines situations rendant inenvisageables toute participation) et pour un même patient au fur et à mesure de sa prise en charge en raison de l'évolution de la maladie tant au plan somatique que psychologique. 
Ces trois points soulignent l'importance de défendre d'une part le principe du caractère non automatique de la participation des patients à sa sécurité et d'autre part du fait que, faire participer ce dernier n'est pas lui demander de se substituer au soignant.

Ce niveau de discernement doit donc faire partie des compétences à acquérir par les professionnels.

Enfin soulignons que l'expertise des patients est un élément qui évolue en fonction de la durée d'évolution de la maladie et de la répétition des traitements. En radiothérapie, les patients répètent la même séance de traitement plusieurs fois et n'ont une connaissance des programmes de soins qu'après un certain délai. On en pourra donc pas impliquer de la même façon un patient en début de prise en charge, que des patients suivis depuis plus longtemps.

\section{Conclusion}

La participation des patients à la gestion de leur propre sécurité est un champ d'étude qui demeure sous-exploité, en radiothérapie comme dans les autres spécialités médicales. Cette étude exploratoire montre que les patients participent effectivement à la gestion des risques, et ce en dépit du faible nombre d'initiatives mises en place à cet effet. Il s'agit d'un axe potentiel d'amélioration de la sécurité des soins qui mérite d'être encouragé et approfondi afin de définir et d'implémenter des situations propices à la participation, cette dernière ne devant en aucun cas être préjudiciable, ni pour les patients, ni pour les professionnels de santé. 


\section{$\underline{\text { Références }}$}

[1] Thouvenin D. Droit à l'information du malade. In Lecourt D, éditeur, Dictionnaire de la pensée médicale, Paris : PUF ; 2004. p.368-73.

[2] François P, Giraud P, Mollo V, Lartigau E. La gestion des risques en radiothérapie. Bull cancer 2010; 97(7):873-80.

[3] Davis RE, Jacklin R, Sevdalis N, Vincent CA. Patient Involvement in Patient Safety: what factors influence patient participation and engagement? Health expect 2007;10:259-67.

[4] Koutantji M, Davis R, Vincent C, Coulter A. The patient's role in patient safety: engaging patients, their representatives, and health professionals. Clin risk 2005;11:99-104.

[5] Buetow S, Elwyn G. Patient safety and patient error. The Lancet 2007;369:158-61.

[6] Agoritsas T, Bovier PA, Perneger TV. Patients reports of undesirable events during hospitalization. J Gen Intern Med 2005;20:922-28.

[7] Lau DH. Patient empowerment; a patient-centred approach to improve care. Hong Kong Med J 2002;8:372-4.

[8] Amalberti R. Sécurité des soins et participation active des patients : promesses ou obstacles? Rencontres HAS 2008. http://www.has-sante.fr/portail/jcms/c_717960/rencontreshas-2008-securite-des-soins-et-participation-active-des-patients-promesses-ou-obstacles.

[9] Amalberti R, Auroy Y, Berwick D, Barach P. Five System Barriers To Achieving

Ultrasafe Health Care. Ann Intern Med 2005;9:756-64.

[10] Coulter A. Paternalism or partnership. BMJ 1999;319:719-20.

[11] Mollo V, Falzon P. Le corps comme objet de l'interaction médecin-patient. Corps 2009;9:69-75.

[12] Lyons M. Should patients have a role in patient safety? A safety engineering view. Qual. Saf. Health Care 2007;16:140-2. 


\section{TABLEAUX}

1. Le patient signale l'oubli d'un cache ou bolus

2. Le patient signale une erreur d'identité

3. Le patient signale qu'un faisceau n'a pas été traité

4. La patiente rectifie la partie du sein à irradier

5. Le patient signale qu'il n'a pas eu sa cure de chimiothérapie irradiosensibilisatrice

6. Le patient a dit qu'il était prêt pour la révision alors qu'il n'avait jamais eu de premier centrage

7. Le patient signale que sa photo n'est pas affichée en salle de traitement (oubli d'allumage des écrans)

8. Le patient signale les erreurs techniques

9. Le patient signale des erreurs de planification

10. Le patient signale lorsqu'il voit le médecin partir et qu'il n'a pas eu sa consultation

11. Le patient compte son nombre de venues à l'hôpital

12. Le patient signale une inadéquation entre le nombre de séances planifiées et le nombre de séances réalisées

13. Le patient signale une différence dans la durée du traitement

14. Le patient écoute de la musique pour se détresser et maintenir sa position

Tableau 1. Les expériences positives (en italique : les signalements d'erreurs et/ou oublis)

Table 1. Positive experiences (italics: descriptions of errors and/or mistakes) 
Le patient et le risque en radiothérapie V.Mollo et al. 21

1. Le patient prend volontairement la place d'un autre patient pour passer plus vite, et reçoit le mauvais traitement

2. Le patient a des projets personnels qui l'amènent à perturber son traitement (départ en vacances, mariage, etc.)

3. Le patient souhaite arrêter son traitement et mobilise l'attention des professionnels qui consacrent moins de temps aux autres patients

Tableau 2. Les expériences négatives

Table 2. Negative experiences 